\title{
Herramienta para el seguimiento del aprendizaje a distancia en alumnos de posgrado. El potencial de Office para realizar envíos personalizados
}

\author{
Estruch-Juan, E. ${ }^{\text {a1 }}$, del Teso, R. ${ }^{\text {a2 }}$, Gómez, E. ${ }^{\text {a3, }}$ Soriano, J. ${ }^{\text {a4 }}$ \\ ${ }^{a}$ ITA (grupo de Ingeniería y Tecnología del Agua). Escuela Técnica Superior de Ingenieros Industriales. Universitat \\ Politècnica de València. \\ a1 maesjua1@ita.upv.es, ${ }^{\mathrm{a} 2}$ rodete@ita.upv.es, ${ }^{\mathrm{a} 3}$ elgosel@ita.upv.es, ${ }^{\mathrm{a} 4}$ jasool@ita.upv.es
}

\begin{abstract}
The amount of online courses offered by ITA has grown significantly in recent years. As a consecuence, the number of students has rosen accordingly. In order to ensure that students adquire the skills required to pass the subjects, self-assessment and a careful supervision by tutors throughout the student's learning process are key aspects. With the growth of the number of students, it is becoming more complex to realise a personalized and detailed follow-up of the students. For this reason, the strategies had to be adapted. Last year, a tool was developed to allow a semi-automated monitorization of students from the Cursosagua online learning platform, where the courses take place. With this tool, tutors can easily detect students' deficiencies and strengths. The tool shows warnings about the tasks performed, the grades obtained, and the time spent in the course. According to these warnings, students receive personalized and automatic messages concerning their performance. This allows the student to perceive a constant and personalized follow-up. This tool makes it easier for tutors to monitor student learning, proving that with this strategy a more continuous and personalized monitoring can be done.
\end{abstract}

Keywords:e-learning, online courses, tutoring, learing monitorization

\section{Resumen}

El número de alumnos en la oferta formativa de docencia online impartida por el ITA ha crecido considerablemente en los últimos años. La autoevaluación y el seguimiento minucioso de los tutores a lo largo del aprendizaje del estudiante son aspectos claves para lograr que adquieran las competencias mínimas requeridas para superar las materias. Con el crecimiento del número de alumnos, el seguimiento personalizado y detallado de los tutores es cada vez más complejo, teniendo que adaptar y modificar las estrategias de seguimiento con el aumento de alumnos. El pasado curso, se desarrolló una herramienta que permite semiautomatizar el seguimiento de los alumnos a partir de los datos extraídos de la Plataforma Cursosagua en la que se desarrollan cada uno de los cursos. Con esta herramienta, los tutores detectan fácilmente las deficiencias y fortalezas de los alumnos. La herramienta muestra advertencias sobre las tareas realizadas, las notas en las evaluaciones, y el tiempo de dedicación, automatizando el envio de mensajes al estudiante en función de las advertencias anteriores. Esto permite que el alumno perciba un seguimiento constante y personalizado, y a su vez facilita la tarea de los tutores, comprobando que con esta estrategia se puede realizar un seguimiento más continuo y personalizado.

Palabras clave: formación a distancia, cursos online, tutorización, seguimiento del aprendizaje 


\section{Introducción}

En 2010 se implementó por primera vez la docencia online en el ITA (grupo de investigación perteneciente al Departamento de Ingeniería Hidráulica y Medio Ambiente de la Universitat Politècnica de València), comenzando por ofertar en modalidad online dos de sus cursos con más demanda presencial. El objetivo era ofrecer esta formación al máximo número de personas posibles, sin desplazamientos, sin importar el lugar de residencia, con la única necesidad de tener un dispositivo con conexión a Internet. La gran acogida de estos dos cursos en los primeros años de docencia a distancia, llevó a desarrollar nuevos contenidos online, y un LMS (Leaning management system) particular pensado específicamente para la docencia de este tipo de cursos, la Plataforma Cursosagua. Tras 10 años, la gran demanda y acogida por parte de los alumnos en cada edición de cursos, ha hecho que la oferta formativa online se haya ido ampliando progresivamente.

En la edición 2016/2017, después de seis años de experiencia en la docencia online, se creó un Plan de Estudios Coordinado de Posgrado, estructurado en un Máster de 660h, un Diploma de 300h y dos Expertos que sumaban 420h, uno de 240h y otro de 180h, y que se amplió en la edición 2018/2019 con otro Experto de 210h. Dicho plan comprende un total de 23 materias que se reparten entre las cinco titulaciones anteriores, siendo algunas de ellas comunes entre varios de los títulos. En total, se ofertan más de 2.500 horas de docencia completamente a distancia, con un creciente número de alumnos curso tras curso, llegando en la edición 2018/2019 a superar las 300 matrículas, de las cuales 57 fueron de títulos de posgrado, y 254 de cursos individuales. Este plan de estudios configura una estructura formativa sólida y con perspectivas de seguir creciendo en el futuro.

Antes de la implementación del Plan de Estudios Coordinado, únicamente existía la opción de matricularse de cursos individuales, pero ya se contaba con un número de alumnos considerable. En la edición 2014/2015 se llegó a contar con 305 matrículas de 224 alumnos diferentes, lo que suponía que cada alumno realizaba de media 1.36 cursos, es decir, la gran mayoría cursaba un único módulo y algunos pocos realizaron ese año dos o más cursos.

Los alumnos matriculados de módulos individuales, cuentan con todo el curso académico para superar los contenidos y evaluaciones de cada materia. La filosofía de estos cursos es que el estudiante pueda inscribirse y comenzar a estudiar los contenidos en cualquier momento, con el objetivo de adaptarse al día a día de técnicos en activo. El alumno puede acceder en cualquier momento a la Plataforma y es el que decide como organizar su propio ritmo de trabajo en función de su disponibilidad. Esta metodología es perfectamente compatible a la hora de realizar módulos individuales, cuya carga lectiva varía entre $30 \mathrm{~h}$ y $60 \mathrm{~h}$, siendo la tasa de abandono menor al $20 \%$, valor inferior a la tasa media de abandono de cursos online situada en un 30\% (Confilegal, 2017).

El seguimiento de estos alumnos es relativamente sencillo, se planifican cinco emisiones de certificados de aprovechamiento a lo largo del curso académico, y las semanas previas a cada emisión se identifican los estudiantes que han finalizado con éxito para poder emitirles el certificado. Se aprovecha la revisión realizada para avisar, al resto de estudiantes que todavía no han acabado, sobre la fecha límite de finalización.

Con la implementación de los títulos de posgrado, se siguió apostando por la flexibilidad de que fuera el propio alumno quien marcara el ritmo de avance en el curso, siendo el desarrollo de cada una de las asignaturas secuencial, es decir, no se habilitan siguiendo un calendario preestablecido, sino que es el mismo alumno quien las va habilitando progresivamente conforme va finalizando las asignaturas previas (Estruch-Juan et al., 2019). Esto asegura que el alumno ha cursado un determinado módulo antes de enfrentarse al siguiente, sin saltar contenidos y siguiendo una estructura donde los módulos previos son 
necesarios para comprender los módulos posteriores. Cada asignatura tiene su correspondiente examen final, planificándose tres periodos de evaluación para realizar estos exámenes. Para presentarse a cada examen, es requisito haber completado todos los contenidos y evaluaciones de la correspondiente materia, pero como cada alumno avanza a su ritmo, en cada convocatoria se especifica a cada uno de ellos a qué exámenes pueden presentarse.

La autoevaluación y el seguimiento minucioso de los tutores a lo largo del aprendizaje del alumno son aspectos claves para lograr que los alumnos adquieran las competencias requeridas para superar los cursos (Pineda et al., 2015). Mantener la motivación del alumnado a lo largo del curso académico es una tarea que está en mano de los tutores, siendo esencial para ello que el alumno perciba una cercanía y un seguimiento detallado de lo que está realizando (Moreira-Segura and Delgadillo-Espinoza, 2015).

Con el crecimiento del número de alumnos, y la implementación de los títulos de posgrado, realizar un seguimiento personalizado y detallado por parte de los tutores es cada vez más complejo, teniendo que adaptar y modificar las estrategias continuamente. No hay que olvidar, que además de la función de seguimiento, los tutores tienen otras funciones de suma importancia, como la función académica, en la que resuelven dudas planteadas por los alumnos y corrigen sus ejercicios, o la función orientadora sobre el funcionamiento del curso, uso de la Plataforma, y cualquier ayuda personalizada que requiera el estudiante para su trayectoria formativa (Fernández-Jiménez et al., 2017). Con el objetivo de mantener una filosofía de cercanía con los alumnos, se garantiza que la respuesta a cualquier consulta será resuelta con la mayor brevedad posible, normalmente en menos de $24 \mathrm{~h}$, lo que conlleva un trabajo considerable de los tutores, más aun si se suman las tareas de seguimiento a realizar.

El pasado curso, se desarrolló una herramienta que permite semiautomatizar el seguimiento de los alumnos a partir de los datos extraídos de la Plataforma Cursosagua y PoliformaT, plataforma de la UPV en la que se realizan los exámenes finales de los títulos de posgrado. La herramienta muestra advertencias sobre las tareas realizadas, las notas en las evaluaciones, y el tiempo de dedicación, automatizando el envío de mensajes al alumno en función de las advertencias anteriores.

En el actual curso académico, se ha implementado el uso de la herramienta desarrollada para realizar el seguimiento de los alumnos. El presente trabajo analiza el impacto que ha tenido la herramienta en los resultados de los estudiantes, y en la dedicación empleada por los tutores a la hora de realizar las diferentes tareas de seguimiento.

\section{Objetivos}

El seguimiento de los alumnos por parte de los tutores es una pieza fundamental para garantizar el éxito en el desarrollo del curso dentro del plazo establecido (Hernández, 2015). La herramienta desarrollada para el seguimiento de los cursos y títulos de posgrado impartidos por el ITA, permite recopilar información detallada del proceso de aprendizaje de los alumnos: grado de cumplimiento de cada materia, calificaciones obtenidas en las diferentes actividades y evaluaciones planteadas, tiempo de dedicación a los recursos de aprendizaje puestos a su disposición... La información recopilada es tratada de manera que se muestran una serie de avisos que permiten detectar posibles deficiencias en el proceso de aprendizaje de cada alumno. La herramienta permite organizar esta información para realizar envíos masivos de advertencias a los alumnos.

Con este trabajo se pretende alcanzar dos objetivos generales vinculados al desarrollo de la herramienta y a la problemática del seguimiento por parte de los tutores: 
- Profundizar de manera detallada en la herramienta desarrollada y en su uso para el seguimiento y envío de avisos a los alumnos. De esta manera, se plantea una primera parte dedicada a explicar el desarrollo de la innovación, que en este caso es la herramienta de seguimiento.

- Analizar el impacto del uso de la herramienta en el día a día de los tutores y en el proceso de aprendizaje de los alumnos. Tras alcanzar el primer objetivo genérico de conocer el funcionamiento y uso de la herramienta, se estará en disposición de alcanzar objetivos más específicos relacionados con el impacto del uso de la herramienta en las tareas a realizar por los tutores, así como en los resultados obtenidos por los alumnos y en su dedicación a cada materia.

A partir de los dos objetivos generales explicados previamente, se busca alcanzar los siguientes objetivos específicos, con la intención de llegar a una serie de conclusiones sobre la herramienta y su uso en el seguimiento de los alumnos:

- Validar la herramienta como instrumento para el seguimiento detallado de los alumnos, especialmente cuando el número de matriculados es relativamente alto. Es fundamental poner la heramienta a prueba para admitirla como válida en el proceso de seguimiento y gestión de los estudiantes. Para ello, se comprobará que funciona correctamente y bajo las premisas con las que se ha desarrollado.

- Comparar los resultados actuales conseguidos usando la herramienta de seguimiento, con los resultados de cursos anteriores. Para poder alcanzar este objetivo es necesario analizar los resultados y dedicación de los alumnos de cursos pasados, donde la estrategia de seguimiento no incluía el uso de la herramienta, así como analizar los resultados y dedicación de los alumnos del presente curso en el que se ha instaurado como elemento de seguimiento. El objetivo es concluir bajo qué estrategia de seguimiento los alumnos obtienen mejores resultados y una dedicación más acorde al tiempo estimado que debe tener cada materia.

- Analizar el tiempo de dedicación de los tutores para realizar el seguimiento y gestión de los alumnos. Si se alcanzan los dos objetivos específicos anteriores, y el tiempo de dedicación de los tutores es el mismo, o incluso inferior que con las estrategias llevadas año anteriores, se podrá concluir que la herramienta es un gran apoyo para ellos, puesto que les permite realizar un seguimiento más detallado y continuo de los alumnos, con una menor dedicación.

\section{Desarrollo de la innovación}

La Plataforma Cursosagua recoge diferente información de cada uno de los alumnos. Un primer apartado de datos personales donde cada estudiante cuenta con un identificativo único y exclusivo. De cada alumno la plataforma registra en qué asignaturas está matriculado, el progreso en cada una de ellas, las evaluaciones realizadas, la calificación obtenida, y el tiempo de dedicación. Dentro de cada uno de estos apartados se especifican registros concretos.

En el caso de las evaluaciones, se registra de cada unidad la nota obtenida en cada una de las evaluaciones planteadas: puntos de control intermedios, test de cada unidad, test final de cada módulo y entrega de ejercicios solicitados (del Teso March et al., 2018). Se ofrece la nota final de cada evaluación y la nota final del curso obtenida en la Plataforma Cursosagua. En el caso de los alumnos matriculados en títulos de posgrado, esta nota representa el 50\% de la nota final, siendo el 50\% restante la nota obtenida en el examen final del curso. En los alumnos matriculados de módulos independientes, la nota de la Plataforma Cursosagua representa la nota del módulo, sin el requisito de realizar un examen final. 
En el caso del tiempo de dedicación, se especifica el tiempo total de conexión a la Plataforma, y se desglosa el tiempo dedicado a cada uno de los recursos de aprendizaje que se ponen a disposición del alumno, así como el tiempo dedicado a cada una de las evaluaciones.

Todos los datos recogidos por la Plataforma Cursosagua pueden exportarse a una hoja de cálculo, tal y como muestra la Fig. 1, con la intención de realizar el tratamiento de datos pertinente para sacar estadísticas o realizar informes de seguimiento. Los exámenes de los alumnos de posgrado se realizan en la plataforma PoliformaT de la Universitat Politècnica de Valencia. Los resultados de éstos también se pueden exportar en una hoja de cálculo en la que se muestra la puntuación de cada alumno para cada examen.

\begin{tabular}{|c|c|c|c|c|c|c|}
\hline \multicolumn{7}{|c|}{ Análisis de redes de agua con EPANET } \\
\hline Desarrollo & $100 \%$ & & & & & \\
\hline \multicolumn{7}{|l|}{ EVALUACION } \\
\hline & Test & Checkpoints & & & & \\
\hline Unidad 1 & 9 & 10 & & & & \\
\hline Unidad 2 & 8 & 7.84 & & & & \\
\hline Unidad 3 & 9 & 9.75 & & & & \\
\hline Unidad 4 & 8 & 8.67 & & & & \\
\hline Unidad 5 & & 10 & & & & \\
\hline Test Final & 9.5 & & & & & \\
\hline \multirow[t]{5}{*}{ Ejercicios } & Ejercicio 1 & 9 & & & & \\
\hline & Ejercicio 2 & 8 & & & & \\
\hline & Ejercicio 3 & 9.5 & & & & \\
\hline & Ejercicio 4 & 9 & & & & \\
\hline & Ejercicio 5 & 9.5 & & & & \\
\hline Nota Final & 9.1 & & & & & \\
\hline \multicolumn{7}{|l|}{ TIEMPOS } \\
\hline & Unidad 1 & Unidad 2 & Unidad 3 & Unidad 4 & Unidad 5 & Total \\
\hline Diapositivas & $0 \mathrm{~h}$ y $35 \mathrm{~min}$. & $6 \mathrm{~h}$ y $34 \mathrm{~min}$. & $8 \mathrm{~h}$ y $21 \mathrm{~min}$. & $13 \mathrm{~h}$ y $25 \mathrm{~min}$. & 7 h y 5 min. & 36 h y 3 min. \\
\hline Checkpoints & $0 \mathrm{~h}$ y $15 \mathrm{~min}$. & $0 \mathrm{~h}$ y $30 \mathrm{~min}$. & $0 \mathrm{~h}$ y $48 \mathrm{~min}$. & $0 \mathrm{~h}$ y $40 \mathrm{~min}$. & $1 \mathrm{~h}$ y $59 \mathrm{~min}$. & $4 \mathrm{~h}$ y $12 \mathrm{~min}$. \\
\hline Test & $0 \mathrm{~h}$ y $50 \mathrm{~min}$. & $0 \mathrm{~h}$ y $50 \mathrm{~min}$. & $0 \mathrm{~h}$ y $50 \mathrm{~min}$. & $0 \mathrm{~h}$ y $50 \mathrm{~min}$. & & $3 \mathrm{~h}$ y $20 \mathrm{~min}$. \\
\hline Descargas & $0 \mathrm{~h}$ y $50 \mathrm{~min}$. & $0 \mathrm{~h}$ y $50 \mathrm{~min}$. & $2 \mathrm{~h}$ y $15 \mathrm{~min}$. & $2 \mathrm{~h}$ y $0 \mathrm{~min}$. & $0 \mathrm{~h}$ y $10 \mathrm{~min}$. & $6 \mathrm{~h}$ y $5 \mathrm{~min}$. \\
\hline Videos & $0 \mathrm{~h}$ y $0 \mathrm{~min}$. & $0 \mathrm{~h}$ y $1 \mathrm{~min}$. & $0 \mathrm{~h}$ y $0 \mathrm{~min}$. & $0 \mathrm{~h}$ y $4 \mathrm{~min}$. & $0 \mathrm{~h}$ y $0 \mathrm{~min}$. & $0 \mathrm{~h}$ y $7 \mathrm{~min}$. \\
\hline Ejercicios & & & & & & $8 \mathrm{~h}$ y $3 \mathrm{~min}$. \\
\hline Test final & & & & & & $1 \mathrm{~h}$ y $40 \mathrm{~min}$. \\
\hline Tiempo Total & & & & & & $59 \mathrm{~h}$ y $31 \mathrm{~min}$. \\
\hline
\end{tabular}

Fig. 1. Exportación de datos de la Plataforma Cursosagua

\subsection{Descripción de la herramienta}

La herramienta desarrollada consiste en una hoja de cálculo Excel programada en Visual Basic Aplication. Permite, a partir de los datos exportados de la Plataforma Cursosagua y de PoliformaT, reordenar y sintetizar la información para realizar el seguimiento de los alumnos. Para ello, la herramienta realiza automáticamente un tratamiento de los datos y ofrece de manera ordenada los alumnos que están realizando cada uno de los módulos y títulos, y su información sobre el desarrollo alcanzado en cada materia.

Se diferencian tres pestañas en la herramienta. Una primera pestaña llamada "Evaluación de Módulos", donde aparece la información del proceso de aprendizaje de los alumnos que están matriculados en módulos individuales. Una segunda pestaña llamada "Evaluación de Títulos", sintetiza la información de los alumnos pertenecientes a títulos de posgrado. Por último, la pestaña "Envíos", permite automatizar el 
envío de correos de seguimiento a partir de los datos recogidos en las otras dos pestañas. A continuación se detallan las distintas acciones llevadas a cabo en cada una de estas pestañas.

\subsubsection{La pestaña "Evaluación de Módulos"}

La pestaña dedicada a la evaluación de módulos requiere únicamente información extraída de la Plataforma Cursosagua, puesto que tal como se ha comentado antes, los alumnos de módulos individuales únicamente cuentan con la evaluación recogida por este LMS. De cada alumno, la herramienta recopila la siguiente información extraída del Excel exportado desde la Plataforma:

- Porcentaje de desarrollo total llevado a cabo

- Cantidad de test de unidad realizados sobre el total

- El número de ejercicios entregados sobre el total

- La nota del test final

- La nota final obtenida

- El tiempo de dedicación total del alumno.

Esta información es la misma que se utilizaba antes del desarrollo de la herramienta para realizar el seguimiento de los alumnos. Pero antes se consultaba alumno por alumno, y ahora se automatiza el proceso, con el consecuente ahorro de tiempo.

La herramienta destaca en color verde aquellos alumnos que han completado todas las actividades y que por lo tanto han finalizado el módulo. Por otro lado, en rojo aparecen aquellos alumnos que no han accedido todavía al curso, tal como puede verse en el ejemplo de la Fig. 2.

La información que aparece en esta pestaña es de gran utilidad para realizar la emisión de certificados a aquellos alumnos que aparecen en verde y que por tanto han acabado el curso. También permite detectar fácilmente alumnos que todavía no han comenzado con el desarrollo del módulo. Pero el gran potencial de la información recogida en esta pestaña es la automatización de envío de correos que se explicará más adelante.

Como información adicional, aparece un aviso para aquellos alumnos que tienen una fecha de finalización determinada. Esto permite realizar un seguimiento exclusivo de este tipo de estudiantes, que son trabajadores matriculados por su empresa y requieren un seguimiento específico. El resto de alumnos tendrán hasta el último día del curso académico para finalizar el curso, por lo que no se específica la fecha en la que deben terminar.

En la Fig. 2 se ve un ejemplo de como la herramienta sintetiza la información del Excel exportado desde la Plataforma (Fig. 1), aportando un resumen del proceso de aprendizaje del estudiante, así como información específica sobre su fecha de finalización. La primera fila de la Fig. 2 se corresponde con el ejemplo de la Fig. 1, en este caso se puede leer de izquierda a derecha que el alumno tiene que finalizar el 19/12/2019, ha desarrollado el 100\% de los contenidos, ha realizado los 4 Test que tiene el curso, siendo la nota del Test Final de un 9,5 y ha entregado los 5 ejercicios solicitados. La nota final del módulo es de 9,1 y el tiempo de dedicación ha sido de $59 \mathrm{~h}$ y $31 \mathrm{~min}$. Cabe destacar que la dedicación de este alumno está acorde con la carga estimada de 60 h que tiene este curso en concreto. Esta información ha sido actualizada el 20/12/2019 a las 12:13. Este caso se corresponde con el seguimiento realizado el 20 de diciembre por parte de los tutores, ya que el día 19/12/2019 era una fecha de finalización para diferentes alumnos de empresa, tal como se puede ver resaltado en gris en la celda Fecha Fin. Además, el color verde identifica que el estudiante ha finalizado con éxito este curso, con lo cual puede emitirse el correspondiente certificado de aprovechamiento. 


\begin{tabular}{|c|c|c|c|c|c|c|c|}
\hline FECHA FIN & $\%$ & TESTS & TESTF & EERCICIOS & NOTA FINAL & TIEMPO DIAP & ACTUALIZACIÓN \\
\hline $19 / 12 / 2019$ & 100 & $4 / 4$ & 9,5 & $5 / 5$ & $\mathbf{9 , 1}$ & $59 \mathrm{~h} \mathrm{y} 31 \mathrm{~min}$. & $20 / 12 / 201912: 13$ \\
\hline & 38,8 & $0 / 4$ & 0 & $0 / 5$ & $\mathbf{0 , 3}$ & $21 \mathrm{~h} \mathrm{y} 11 \mathrm{~min}$. & $12 / 03 / 202013: 22$ \\
\hline & 0 & $0 / 4$ & 0 & $0 / 5$ & 0 & $0 \mathrm{~h} \mathrm{y} 0 \mathrm{~min}$. & $12 / 03 / 202013: 22$ \\
\hline & 100 & $4 / 4$ & 8 & $5 / 5$ & $\mathbf{8 , 7}$ & $53 \mathrm{~h} \mathrm{y} 11 \mathrm{~min}$. & $12 / 03 / 202013: 22$ \\
\hline
\end{tabular}

Fig. 2. Herramienta para el seguimiento de alumnos de módulos Cursosagua

\subsubsection{La pestaña "Evaluación de Títulos"}

En la pestaña dedicada a la evaluación de títulos, la herramienta clasifica los alumnos en función del título de posgrado en el que está matriculado. De cada alumno rellena automáticamente la información de cada una de las asignaturas que va realizando. Esta información se puede dividir en dos partes, por un lado la información extraída de la Plataforma Cursosagua, indicando el desarrollo del módulo, las actividades que lleva desarrolladas y la nota final de esta parte, que representa el $50 \%$ de la nota de la materia. Por otro lado la información extraída del examen final realizado en PoliformaT, registrando la convocatoria en la que ha realizado el examen y su nota. Toda esta información se puede leer de manera ordenada de izquierda a derecha en el ejemplo la Fig. 3. A partir de la nota de la Plataforma y de la nota del examen final, muestra la nota definitiva de la materia.

\begin{tabular}{|c|c|c|c|c|c|c|c|c|c|c|c|}
\hline \multicolumn{6}{|c|}{ HIDRÁULICA } & \multicolumn{6}{|c|}{ HIDROLOGÍA } \\
\hline$\%$ & FALTA & NOTACA & CONVOC & NOTA EX & NOTA MÓD & $\%$ & FALTA & NOTACA & CONVOC & NOTA EX & NOTA MÓD \\
\hline 100 & $6 / 6-10-0 / 0$ & 9,2 & 1 & 7,75 & 8,48 & 100 & $6 / 6-9-2 / 2$ & 8,7 & 1 & 6,13 & 7,42 \\
\hline 100 & $6 / 6-10-0 / 0$ & 9,2 & 1 & 5,88 & 7,54 & 100 & $6 / 6-10-2 / 2$ & 9,3 & 1 & 8,42 & 8,86 \\
\hline 100 & $6 / 6-10-0 / 0$ & 10 & 1 & 9 & 9,50 & 100 & $6 / 6-10-2 / 2$ & 9,9 & 1 & 10 & 9,95 \\
\hline 100 & $6 / 6-10-0 / 0$ & 9,8 & 1 & 9,5 & 9,65 & 100 & $6 / 6-10-2 / 2$ & 9,9 & 1 & 6,5 & 8,20 \\
\hline 100 & $6 / 6-10-0 / 0$ & 9,9 & & & & 7,8 & $0 / 6-0-0 / 2$ & & & & \\
\hline 100 & $6 / 6-10-0 / 0$ & 9,7 & 0 & 7 & 8,35 & 100 & $6 / 6-10-2 / 2$ & 9,2 & 1 & 7,25 & 8,23 \\
\hline 100 & $6 / 6-9-0 / 0$ & 9,4 & 0 & 10 & 9,70 & 51,4 & $2 / 6-0-0 / 2$ & & & & \\
\hline 100 & $6 / 6-9-0 / 0$ & 9,1 & 1 & 10 & 9,55 & 100 & $6 / 6-9-2 / 2$ & 9,3 & 1 & 9 & 9,15 \\
\hline 100 & $6 / 6-10-0 / 0$ & 9,7 & 0 & 10 & 9,85 & 100 & $6 / 6-10-2 / 2$ & 9,8 & & & \\
\hline 100 & $6 / 6-10-0 / 0$ & 9,6 & 0 & 9 & 9,30 & 100 & $6 / 6-10-2 / 2$ & 9,8 & 1 & 8,75 & 9,28 \\
\hline 100 & $6 / 6-10-0 / 0$ & 9,4 & 0 & 8 & 8,70 & 100 & $6 / 6-10-2 / 2$ & 9,8 & 1 & 3,96 & 6,88 \\
\hline 100 & $6 / 6-10-0 / 0$ & 9,5 & 0 & 10 & 9,75 & 100 & $0 / 6-0-0 / 2$ & & & & \\
\hline 100 & $6 / 6-10-0 / 0$ & 9,6 & 0 & 5,75 & 7,68 & 100 & $6 / 6-10-2 / 2$ & 9,5 & 0 & 5,25 & 7,38 \\
\hline 100 & $6 / 6-10-0 / 0$ & 9,5 & 0 & 9 & 9,25 & 100 & $6 / 6-9-2 / 2$ & 9,5 & & & \\
\hline 100 & $6 / 6-9-0 / 0$ & 8,3 & 1 & 10 & 9,15 & 100 & $6 / 6-8-2 / 2$ & 8,4 & 1 & 7,75 & 8,08 \\
\hline 100 & $6 / 6-10-0 / 0$ & 9,6 & 0 & 10 & 9,80 & 100 & $6 / 6-10-2 / 2$ & 9,7 & 1 & 3,25 & 6,48 \\
\hline
\end{tabular}

Fig. 3 Herramienta para el seguimiento de los alumnos de posgrado Cursosagua

El código de colores establecido permite conocer de una manera rápida cual es el estado de los estudiantes matriculados en los títulos de posgrado. Los alumnos con la información de una materia en verde, son alumnos que han finalizado por completo y han superado esa asignatura. Los alumnos en amarillo, son alumnos que han finalizado los contenidos en la Plataforma, pero que todavía no han realizado el examen final. En rojo aparecen los alumnos que han suspendido alguna de las partes. La nota mínima tanto en la Plataforma como en el examen debe ser de 5, por lo que se marca en rojo los alumnos que no llegan a este mínimo. Por último, en gris aparecen los módulos que son convalidados debido a que han sido superados en ediciones previas. Se corresponde con alumnos repetidores o que han realizado algún otro título del plan de estudios coordinado que incluye esta asignatura, y por tanto se convalida automáticamente.

Si el alumno no ha comenzado con el desarrollo de una materia simplemente aparece en blanco y sin ningún contenido. Si habiéndola comenzado, todavía no ha finalizado por completo, el color es blanco pero aparece la información del desarrollo llevado a cabo y del número de actividades realizadas. Una vez culminadas todas las materias, la herramienta calcula la nota final del título, ponderando la carga lectiva de cada módulo con su resultado obtenido. 


\subsubsection{La pestaña "Envíos"}

Una vez han sido migrados los datos a la herramienta, se pueden preparar varias acciones de seguimiento de los alumnos a partir de la configuración de la información que aparece en la tercera pestaña de la herramienta, la pestaña "Envíos". Las acciones son distintas en función de si el alumno está realizando módulos o si es un alumno de posgrado, pero el objetivo es el mismo: enviar automáticamente información a cada estudiante sobre su avance en la materia, con el objetivo de ofrecerle un feedback acerca de su dedicación y resultados.

La herramienta permite preparar automáticamente los archivos necesarios para realizar el envío de correos mediante la opción de correspondencia de Microsoft Word. Los envíos a realizar consisten en envíos de seguimiento para alumnos de módulos y títulos, envíos de convocatorias de examen y envíos de los resultados obtenidos para los alumnos de posgrado. En el siguiente apartado de revisan detalladamente.

Estos envíos automáticos ya se realizaban antes de crear la herramienta, pero suponían mucho trabajo para migrar los datos de las plataformas y representarlos en el formato adecuado para realizar la correspondencia de Word. La nueva herramienta ha conseguido reducir el tiempo de horas a pocos minutos.

\subsection{Usos de la herramienta}

Tal y como se recomienda en algunos manuales (Escuela de Administración Pública, 2014), en el seguimiento de la docencia online se debe prestablecer posibles escenario (estudiantes retrasados en su avance, evaluaciones favorables y desfavorables, recordatorios de fechas importantes...) Tras los años de experiencia en la docencia online impartida por el ITA, diferentes situaciones y casuísticas se repiten cada año académico. Para afrontar estos casos, se tienen preparados una serie de textos que contemplan las posibles situaciones en las que se encuentran los alumnos. Por ejemplo, un alumno de módulo, puede estar a punto de finalizar el módulo, estar empezando, o ni siquiera haber accedido a la plataforma Cursosagua para empezar con la formación. Los textos preparados están escritos teniendo en cuenta la comunicación asertiva y tratan de motivar al alumno para finalizar con éxito el curso (Escuela de Administración Pública, 2014).

La preparación de los datos necesarios para realizar el envío automático de correos, junto a los textos predefinidos para cada caso contemplado, permite realizar el envío masivo de correos con la situación particular de cada alumno. De este modo, la herramienta permite enviar a cada alumno su seguimiento de forma personalizada, viable (dado el elevado número de alumno) y sin perder la proximidad con el alumno.

A continuación se detallan las distintas acciones de seguimiento llevadas a cabo, y cómo influye la herramienta desarrollada en cada caso.

\subsubsection{Seguimiento de los alumnos de módulos}

A lo largo del curso se revisa de forma regular el avance de los alumnos de módulo y se les envía un correo indicándoles su desarrollo hasta el momento, así como las tareas que tienen pendientes. De este modo, se le transmite al alumno una cercanía y atención por parte de los tutores sobre su progreso, y al mismo tiempo, se le recuerda la fecha límite, con el fin de que no se deje el curso para el último momento. 
La herramienta detecta automáticamente si el avance del alumno es el esperado, o no, en función del desarrollo en el módulo y de la cercanía de la fecha de finalización. A partir de aquí se aplica un texto a enviar en el correo u otro.

A continuación se muestra un ejemplo de los textos enviados de manera semiautomática a los alumnos cuando se les realiza el seguimiento. En cursiva aparecen los campos que se rellenarían automáticamente a partir de la correspondencia de Word, y de los datos obtenidos con la herramienta de seguimiento. Este ejemplo en concreto se corresponde con un alumno de módulo cuyo desarrollo es adecuado:

Buenos días NOMBRE DEL ALUMNO,

Te recordamos que la fecha límite para finalizar el curso NOMBRE DEL CURSO es el próximo $D D / M M / A A A A$. Revisando el progreso de los alumnos, hemos observado que en tu caso llevas desarrollado el \% DESARROLLADO del contenido del curso. Tu avance es bueno, pero no pierdas de vista la fecha límite para finalizar el curso.

Ante cualquier consulta no dudes en ponerte en contacto con nosotros.

Un saludo

Al tener alumnos con distintas fechas de finalización, la herramienta permite particularizar el seguimiento únicamente para aquellos alumnos cuya fecha de finalización está cercana o para todos los alumnos, independientemente de cuando finalicen.

Estas tareas se realizaban anteriormente de forma manual. Debido al elevado número de alumnos eran muy costosas en tiempo y contaban con una alta probabilidad de fallo humano.

\subsubsection{Seguimiento de los alumnos de títulos de posgrado}

El seguimiento de los alumnos que están cursando títulos de posgrado es más pormenorizado. Se trata de alumnos que realizan un número elevado de asignaturas, entre 4 y 13 en función del título. Para superar con éxito el curso es preciso que mantengan un estudio constante, ya que de lo contrario no podrán finalizar la formación a tiempo. Tal y como se ha comentado en la introducción, la formación es secuencial, siendo el alumno quien va habilitando los contenidos y las asignaturas conforme avanza en el temario, de ahí la importancia de mantener una constancia en el estudio, y no dejarlo para el último momento.

Además de la ayuda visual que supone el código de colores explicado anteriormente, se dispone de tres tipos de envíos semiautomatizados de correos que permiten a los tutores comunicar a los alumnos su desempeño en relación al título que están cursando:

El primero de ellos, sería el equivalente al de los alumnos de módulos. Se trata de evaluar el avance del alumno y comunicárselo. Para ello, se compara el avance de cada estudiante hasta el momento, con el avance esperado de acuerdo con el calendario estimado recogido en la guía docente.

Cada título cuenta con un calendario estimativo que sugiere el tiempo óptimo de dedicación a cada asignatura para poder culminar con éxito y sin prisas de última hora. Se cuenta con alumnos con mayor disponibilidad temporal y/o motivación que van más avanzados respecto al calendario propuesto, y alumnos que van más retrasados, ya sea por motivos laborales, falta de dedicación o una incorporación más tardía al título. La herramienta detecta los alumnos que llevan un ritmo acorde al establecido en el calendario propuesto, y aquellos que van retrasados.

Así, mediante la herramienta desarrollada, se envía a cada estudiante un correo que incluye una tabla en la que se muestran todas las asignaturas de las que dispone el título, y el porcentaje de realización que lleva 
el alumno en cada una de ellas, tal y como se muestra en la Tabla 1. En el caso de que el desarrollo del alumno esté en línea con el calendario propuesto, o incluso esté más avanzado, se le felicita por su desempeño y se le anima a que siga así. En el caso de que el alumno vaya más retrasado, se le indica cuál es el módulo que debería de estar desarrollando y se le motiva para que aumente su dedicación y pueda superar a tiempo el título.

Tabla 1. Desarrollo de un alumno de Máster

\begin{tabular}{|c|c|c|c|c|}
\hline \multicolumn{5}{|c|}{ Área de Hidráulica } \\
\hline Hidráulica & Hidrología & Válvulas & & Contadores \\
\hline $100 \%$ & $100 \%$ & $100 \%$ & & $100 \%$ \\
\hline \multicolumn{5}{|c|}{ Área de Modelación } \\
\hline EPANET & Dime & & SWMM & ALLIEVI \\
\hline $100 \%$ & & & $94 \%$ & $0 \%$ \\
\hline \multicolumn{5}{|c|}{ Área de Gestión } \\
\hline Indicadores & & & Pérdidas & Gestión de la Demanda \\
\hline \multirow[t]{3}{*}{$0 \%$} & & & $0 \%$ & $0 \%$ \\
\hline & De & el Máster: & $60 \%$ & \\
\hline & De & sperado: & $92 \%$ & \\
\hline
\end{tabular}

El segundo envío automatizado para alumnos de posgrado, es el envío de la convocatoria de exámenes. Cabe recordar, que a lo largo del curso académico hay tres convocatorias en las que los alumnos pueden presentarse a todas las asignaturas que haya completado hasta el momento. Así, cada alumno se presenta a un número de asignaturas distinto, en función de su avance. Unas semanas antes de los exámenes se les envía un correo personalizado en el que se les informa de las asignaturas que podrán rendir.

La nueva herramienta lleva implementada una función que permite recopilar, para cada alumno, las asignaturas a las que puede presentarse, las que ya ha aprobado en convocatorias anteriores, las convalidadas si es el caso, y si debe recuperar algún examen suspendido en convocatorias previas (Tabla 2). Si en el momento del envío está a punto de finalizar una asignatura, se le anima a completarla lo antes posible para poderse presentar a su examen y tener tiempo de revisar el resto de materias. En caso contrario, se le recomienda estudiar los temarios del resto de módulos a los que se presentará en la convocatoria.

Tabla 2. Notificación de las asignaturas a presentarse en una convocatoria de exámenes

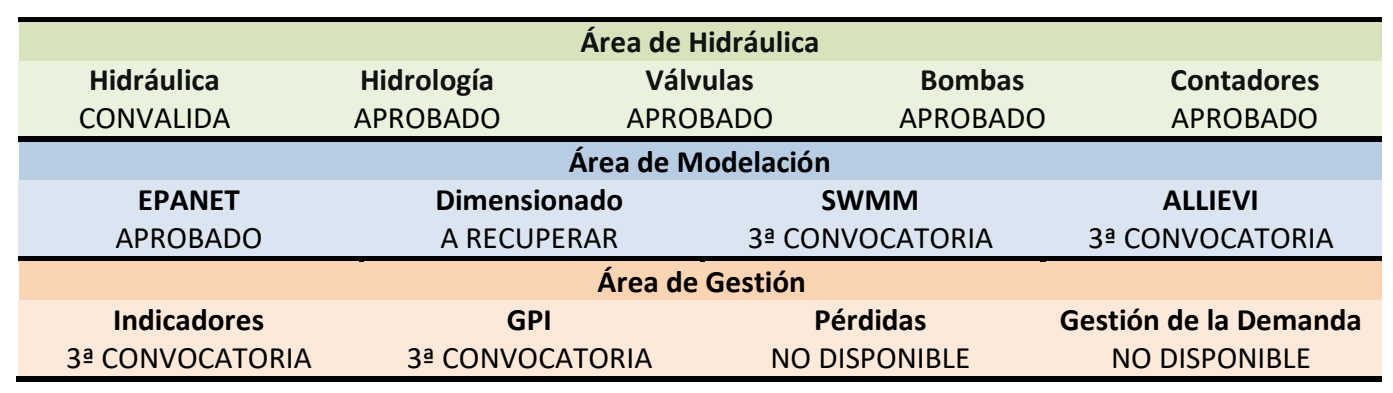

El tercer y último tipo de envío semiautomatizado para alumnos de posgrado, consiste en un correo con los resultados obtenidos tras los exámenes y al finalizar el curso. En éste, se les informa de la nota final obtenida en cada módulo, compuesta a partir de la nota obtenida en el examen y de la nota obtenida en la evaluación continua realizada en la Plataforma. Al final de curso se les facilita también la nota media del título. En función de sus resultados, se les felicita por haber conseguido finalizar con éxito el 
título y se les comentan los pasos a seguir para obtener el título. Si no han podido superar el título, se les informa de las distintas opciones de las que disponen. La Tabla 3 muestra los resultados enviados al alumno al finalizar el curso.

Tabla 3. Desglose de calificaciones de un alumno.

\begin{tabular}{|c|c|c|c|c|c|c|c|c|c|c|c|c|c|c|}
\hline \multicolumn{3}{|c|}{ Hidráulica } & \multicolumn{3}{|c|}{ Hidrología } & \multicolumn{3}{|c|}{ Válvulas } & \multicolumn{3}{|c|}{ Bombas } & \multicolumn{3}{|c|}{ Contadores } \\
\hline Examen & Curso & Nota & Examen & n Curso & Nota & Examen & Curso & Nota & Examen & Curso & Nota & Examen & Curso & Nota \\
\hline 6,6 & 9,3 & 8,0 & 6,8 & 7,9 & 7,3 & 6,0 & 8,5 & 8,0 & 6,9 & 7,9 & 7,4 & 7,7 & 10 & 8,8 \\
\hline \multicolumn{4}{|c|}{ EPANET } & \multicolumn{3}{|c|}{ Dimensionado } & \multicolumn{4}{|c|}{ SWMM } & \multicolumn{4}{|c|}{ ALLIEVI } \\
\hline Examen & Curso & & Jota & Examen & Curso & Nota & & amen & Curso & Nota & Exa & nen & arso & Nota \\
\hline 7,3 & 8,9 & & 8,1 & 7,8 & 8,7 & 8,2 & & 10 & 9,7 & 9,9 & & 8 & 1 & 8,9 \\
\hline \multicolumn{4}{|c|}{ Indicadores } & \multicolumn{3}{|c|}{ GPI } & \multicolumn{4}{|c|}{ Pérdidas } & \multicolumn{4}{|c|}{ Gestión de la Demanda } \\
\hline Examen & Curso & & Nota & xamen & Curso & Nota & Exar & amen & Curso & Nota & Exa & men & arso & Nota \\
\hline 6,3 & 8,3 & & 7,3 & 9,1 & 9,0 & 9,0 & & 7,5 & 9,8 & 8,7 & & 0 & 4 & 9,7 \\
\hline
\end{tabular}

Para cada una de las acciones de seguimiento explicadas en este apartado, se dispone de textos preestablecidos que cambian en función de los escenarios que puedan darse en cada caso. El texto enviado a cada alumno dependerá de su casuística particular. En el caso de los seguimientos, se detalla a cada alumno su situación, explicándole las tareas que le quedan para finalizar por completo (ejercicios, test, etc.) El texto cambia en función de si el alumno va retrasado o sigue la programación prevista. La opción de correspondencia de Word permite que a partir de los textos preestablecidos, y de los datos que prepara la herramienta, el envío de correos sea rápido, particularizado para cada alumno y apropiado para el escenario en el que se encuentre el alumno.

\section{Resultados}

La herramienta presentada se ha programado con el fin de mejorar la tutorización de los más de 300 alumnos anuales con los que cuenta la formación Cursosagua, entre alumnos de módulos y títulos de posgrado. Los resultados obtenidos se han alcanzado comparando la información registrada de ediciones pasadas con la información de la actual edición 19/20 que comenzó en septiembre de 2019. Una vez finalizado por completo el presente curso académico, en julio de 2020, se obtendrán resultados más sólidos, que refuercen lo que se expone en este apartado.

Tras el análisis de la información recopilada hasta el momento, se ha detectado que el mayor cambio se produce en el seguimiento de los alumnos de posgrado. El alumnado matriculado en módulos independientes sigue la misma tendencia en cuanto a dedicación y resultados que en ediciones posteriores. Sin embargo, es destacable que el tiempo empleado por parte de los tutores para realizar las tareas de seguimiento de estos alumnos se ha reducido considerablemente. A continuación se exponen los resultados más significativos sobre alumnos de posgrado, y sobre la dedicación de los tutores a realizar tareas de seguimiento.

\subsection{Dedicación de los alumnos de posgrado}

Se ha observado que en las dos últimas ediciones, $17 / 18$ y 18/19, la tasa de seguimiento del calendario propuesto a los alumnos descendía entre la primera y la segunda convocatoria de exámenes, y aumentaba considerablemente en la tercera y última tanda de exámenes, tal como puede verse en la Fig. 4. 


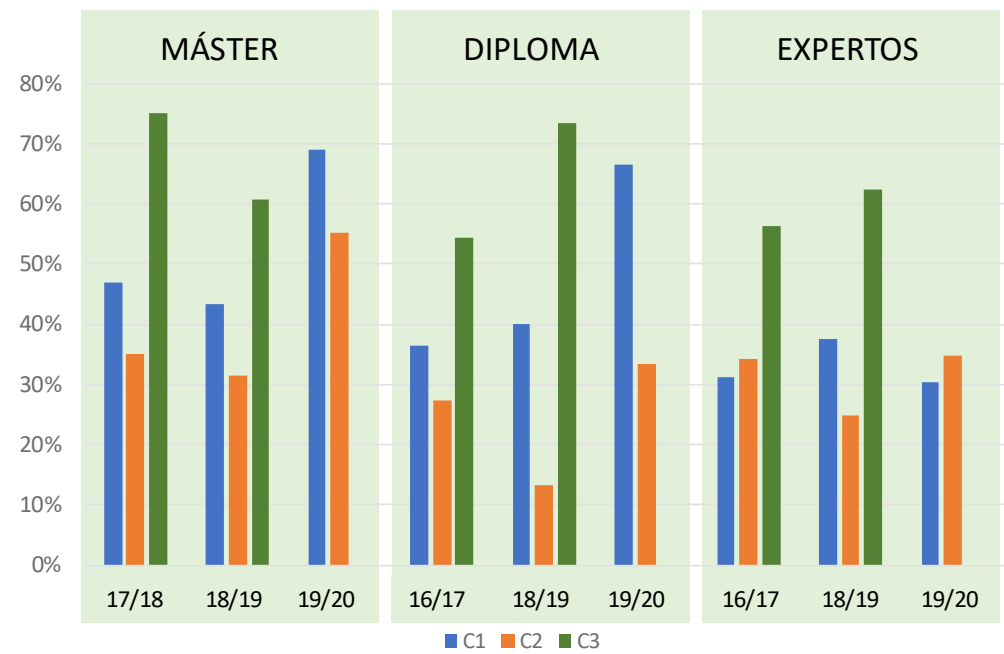

Fig. 4 Tasa de seguimiento del calendario propuesto para alumnos de posgrado

Esto refleja que los alumnos comienzan el curso siguiendo un estudio relativamente constante, alrededor del 40\% cumplen la planificación prevista, pero después desciende esta dedicación. Quizá el motivo sea la confianza de los resultados obtenidos en la primera convocatoria de exámenes, siempre por encima del 8,5 de media.

En la segunda convocatoria, el ratio de desempeño baja a valores incluso por debajo del $30 \%$ de la planificación prevista, y se incrementa en la última convocatoria hasta valores entorno al $60-70 \%$, siendo ésta la tasa de éxito de los títulos. Esta tendencia, deja ver que es en el último tercio del curso donde los alumnos más tiempo dedican a completar los contenidos, en lugar de realizar un estudio lo más continuado posible durante todo el curso académico.

A falta de realizar la última convocatoria de exámenes en la presente edición 19/20, se puede ver en la Fig. 4 como tras implementar el uso de la herramienta de seguimiento, en la primera convocatoria los alumnos se han acercado más al rendimiento esperado, con una tasa próxima al $70 \%$ en el Máster y Diploma y mucho más baja en el caso de los Expertos. La segunda convocatoria, pese que en esta ocasión también ha sufrido un descenso de la dedicación respecto a la primera, sigue estando por encima de ediciones pasadas, especialmente en el Máster. La tendencia indica que los alumnos están siguiendo un ritmo de estudio más constante, y no tan concentrado en las últimas semanas del curso. A falta de la última convocatoria de exámenes, se espera que la tasa de éxito final sea superior a la de ediciones precedentes.

\subsection{Calificaciones de los alumnos de posgrado}

Se ha comprobado que además de ser la convocatoria con más exámenes realizados, la tercera convocatoria es la que peores resultados tiene, tal y como se observa en la Tabla 4. Aunque los resultados se pueden considerar buenos, con calificaciones medias por encima del 8,5 en todas las convocatorias, es esta tercera convocatoria la que peor nota obtiene, con una nota media en todos los títulos y ediciones inferior a la nota media final. 
Estruch-Juan, E., del Teso, R., Gómez, E., Soriano, J.

Tabla 4.Calificaciones medias obtenidas por los alumnos de posgrado en cada convocatoria de exámenes

\begin{tabular}{|c|c|c|c|c|c|c|c|c|c|}
\cline { 2 - 10 } \multicolumn{1}{c|}{} & \multicolumn{3}{c|}{$\mathbf{1 7 / 1 8}$} & \multicolumn{3}{c|}{$\mathbf{1 8 / 1 9}$} & \multicolumn{3}{c|}{$\mathbf{1 9 / 2 0}$} \\
\cline { 2 - 10 } \multicolumn{1}{c|}{} & Máster & Diploma & Expertos & Máster & Diploma & Expertos & Máster & Diploma & Expertos \\
\hline Convocatoria 1 & 9,1 & 9,2 & 8,7 & 8,9 & 9,1 & 9 & 8,9 & 9,4 & 8,7 \\
\hline Convocatoria 2 & 8,9 & 9,2 & 9 & 8,9 & 9,5 & 9,1 & - & - & - \\
\hline Convocatoria 3 & 8,7 & 9 & 8,6 & 8,8 & 9,1 & 8,8 & - & - & - \\
\hline Nota media final & $\mathbf{8 , 8 4}$ & $\mathbf{9 , 1 1}$ & $\mathbf{8 , 7 0}$ & $\mathbf{8 , 8 7}$ & $\mathbf{9 , 1 2}$ & $\mathbf{8 , 9 0}$ & - & - & - \\
\hline
\end{tabular}

Si bien de momento únicamente se cuenta con las calificaciones de la primera convocatoria de la edición 19/20 (se ha convocado la segunda convocatoria pero todavía no ha finalizado) se ha visto que los resultados son similares a los obtenidos en ediciones pasadas. Sin embargo, se espera que la dedicación más distribuida en el tiempo lleve en la tercera convocatoria a mejorar los resultados respecto a otras ediciones, sin ser necesariamente esta convocatoria la que tenga notas por debajo de la media.

\subsection{Tareas y dedicación de los tutores}

Aunque la tarea y dedicación de los tutores es difícilmente medible con números y estadísticas, se conoce con garantía los cambios que la herramienta ha producido en las tareas que día a día tienen que realizar. En próximos cursos académicos, se prevé implementar encuestas cualitativas que recojan las impresiones de los tutores para determinar con mayor precisión la reducción temporal que han supuesto estas mejoras. A continuación se describen los cambios y mejoras que los tutores han percibido en el presente curso académico en su tarea de seguimiento.

El tiempo empleado para revisar los alumnos que han finalizado los módulos, y así emitir sus correspondientes certificados de aprovechamiento, se ha reducido a unos pocos minutos. Básicamente se reduce al tiempo que se necesita en exportar los datos de la Plataforma e importarlos en la herramienta. Con el color verde se detecta rápidamente qué alumnos han finalizado, y cuál es su nota final. Esta tarea conllevaba un tiempo de dedicación anteriormente de unas tres horas por cada emisión de certificados, ya que había que revisar alumno por alumno.

Uno de los resultados más destacables se produce en el seguimiento de los alumnos de títulos de posgrado. La herramienta facilita enormemente su seguimiento así como la convocatoria de exámenes, reduciendo el tiempo de dedicación de los tutores al automatizar el envío de correos personalizados a cada alumno. En dicho correo se indica a qué asignaturas pueden presentarse, fechas de la convocatoria... tarea que antes se realizaba manualmente alumno por alumno.

También se ha reducido notoriamente el tiempo dedicado a consultar las notas de la Plataforma Cursosagua y del examen realizado en PoliformaT, para posteriormente trasladarlas manualmente a una hoja Excel en el que se calculaba y registraba la nota de cada materia. En esta edición, la migración automática de los resultados de las dos plataformas reduce el tiempo dedicado a esta tarea a unos pocos segundos, y además permite minimizar los errores humanos. Esta tarea de unos pocos segundos, antes implicaba una jornada laboral completa en la que se implicaban dos tutores para rellenar todos los datos. La facilidad que presenta la herramienta para organizar los datos de los envíos en el formato adecuado para enviar los correos a través de Correspondencia de Word, ha eliminado el trabajo de reorganizar los datos que anteriormente lo realizaban de forma manual los tutores.

Por último, el seguimiento planteado con el uso de la herramienta desarrollada, ha permitido una mayor facilidad para detectar alumnos que se quedan rezagados y hacerles un seguimiento personalizado. Al reducirse el tiempo empleado en realizar gestiones mecánicas: pasar notas desde las plataformas, consultar uno a uno los alumnos finalizados, enviar manualmente correos personalizados..., se dedica una 
mayor atención al alumno. Esto ha conllevado la disminución del tiempo de respuesta a consultas, convirtiéndose la interacción con el alumno en un proceso más fluido. Al realizar un seguimiento más cercano, el alumno adquiere más confianza con el tutor y participa más, detectando en la actual edición un aumento de consultas respecto a ediciones pasadas.

\section{Conclusiones}

El uso de una herramienta desarrollada a partir de una hoja Excel programada, junto al potencial de la opción de correspondencias de Word, ha permitido mejorar el seguimiento de los alumnos matriculados en módulos y títulos de posgrado del Plan de Estudios Coordinado del ITA. La automatización del envío de correos, ha permitido realizar un seguimiento más personalizado y constante de los alumnos, en especial, los de posgrado. Este hecho se ha traducido en una dedicación más constante de los alumnos desde septiembre hasta el momento. La tasa de abandono en estudios de Máster en universidades públicas no presenciales es del 36,9\% (Ministerio de Ciencia Innovación y Universidades, 2019), mientras que en los estudios de posgrado ofertados por el ITA la tasa de abandono media actual se sitúa en un $36 \%$. Se espera que con el seguimiento realizado a través de la herramienta, la tasa de abandono en el presente curso disminuya, al menos ésta es la tendencia en las dos primeras convocatorias de examen realizadas.

El tiempo de dedicación de los tutores para realizar el envío de correos de seguimiento se ha visto reducido notoriamente al automatizar esta tarea. Por otro lado, la automatización de la exportación de notas, ha reducido los posibles errores que conllevaba la exportación manual realizada anteriormente.

En definitiva, haciendo uso de dos de los programas más utilizados a nivel mundial, como son Excel y Word, se ha optimizado las tareas de los tutores, consiguiendo una dedicación más constante de los alumnos al estudio de las materias, lo que se espera que contribuya con una reducción de la tasa de abandono a final de curso.

\section{Referencias}

CAREAGA BUTTER, B. (2013). "La tutoría virtual. Un rol emergente en el nuevo paradigma educativo". Blog educación. $\quad$ https://www.ucsc.cl/blogs-academicos/la-tutoria-virtual-un-rol-emergente-en-el-nuevo-paradigmaeducativo/.

CONFILEGAL. (2017). La formación "on line" no funciona del todo bien: $30 \%$ de abandono en los cursos de pago y $90 \%$ en los gratuitos. Confilegal. Retrieved from https://confilegal.com/20170614-la-formacion-on-line-no-funcionadel-bien-30-abandono-los- cursos-pago-90-los-gratuitos/

DEL TESO MARCH, R., ESTRUCH JUAN, E., GÓMEZ SELLÉS, E. AND SORIANO OLIVARES, J. (2018). "Sistema de evaluación para la formación a distancia de profesionales". INRED 2018. Congreso Nacional de Innovación Educativa y de Docencia en Red, pp. 875-888, https://doi.org/10.4995/inred2018.2018.8748.

ESCUELA DE ADMINISTRACIÓN PÚBLICA. (2014). "Manual de buenas prácticas del profesorado tutor de Teleformación", https://efiapmurcia.carm.es/web/pagina?IDCONTENIDO=48300\&IDTIPO=100\&RASTRO=c\$m2813,5280.

ESTRUCH-JUAN, E., DEL TESO MARCH, R., GÓMEZ SELLÉS, E. AND SORIANO OLIVARES, J. (2019). "Metodología docente para la enseñanza técnica online. La experiencia Cursosagua." Proceedings INNODOCT/19. International Conference on Innovation, Documentation and Education., Valencia, Spain: Editorial Universitat Politècnica de València. ISBN: 978-84-9048-799-0 
FERNÁNDEZ-JIMÉNEZ, M.Á., MENA-RODRÍGUEZ, E. AND TÓJAR-HURTADO, J.C. (2017). "Funciones de la tutoría en e-learning: Estudio mixto de los roles del tutor online". Revista de Investigación Educativa 35(2): 409-426, https://doi.org/10.6018/rie.35.2.273271.

HERNÁNDEZ, P. (2015). "Funciones de la tutoría virtual". http://www.monografias.com/trabajos-pdf/funcionestutoria-virtual/funciones-tutoria-virtual.pdf.

MINISTERIO DE CIENCIA INNOVACIÓN Y UNIVERSIDADES. (2019). Datos y Cifras del Sistema Universitario Español 2018-2019. Sistema Universitario Español. https://cpage.mpr.gob.es/.

MOREIRA-SEGURA, C. AND DELGADILLO-ESPINOZA, B. (2015). "La virtualidad en los procesos educativos: reflexiones teóricas sobre su implementación.” Tecnología en Marcha 28(1). ISSN 0379-3982

PINEDA, P., VALDIVIA, P. AND CIRASO, A. (2015). “Actividades en Moodle: Manual de buenas prácticas pedagógicas", https://ddd.uab.cat/pub/estudis/2016/149926/Moodle buenas practicas.pdf. 